\title{
Upregulated Claudin-1 Expression Promotes Colitis-associated Cancer by promoting $\beta$-Catenin phosphorylation and activation in Notch/p-AKT Dependent Manner
}

\author{
Saiprasad Gowrikumar ${ }^{1}$, Rizwan Ahmad ${ }^{1}$, Srijayaprakash Babu Uppada ${ }^{1}$, Mary K \\ Washington ${ }^{4}$, Chanjuan $\mathrm{Shi}^{4}$, Amar B. Singh ${ }^{1,2,3}$, and Punita Dhawan ${ }^{1,2,3}$ \\ ${ }^{1}$ VA Nebraska-Western lowa Health Care System, Omaha, NE \\ ${ }^{2}$ Department of Biochemistry and Molecular Biology, University of Nebraska Medical Center, \\ Omaha, NE \\ ${ }^{3}$ Buffet Cancer Center, University of Nebraska Medical Center, Omaha, NE \\ ${ }^{4}$ Department of Pathology, Vanderbilt University Medical Center, Nashville, TN.
}

\begin{abstract}
In IBD patients, integration between a hyper-activated immune system and epithelial cell plasticity underlies colon cancer development. However, molecular regulation of such a circuity remains undefined. Claudin-1(Cld-1), a tight-junction integral protein deregulation alters colonic epithelial cell (CEC) differentiation, and promotes colitis severity while impairing colitis-associated injury/ repair. Tumorigenesis is a product of an unregulated wound healing process and therefore we postulated that upregulated Cld-1 levels render IBD patients susceptible to the colitis-associated cancer (CAC). Villin Cld-1 mice is used to carryout overexpressed studies in mice. The role of deregulated Cld-1 expression in CAC and underlying mechanism using a well-constructed study scheme and mouse models of DSS colitis/recovery and CAC. Using an inclusive investigative scheme, we here report that upregulated Cld-1 expression promotes susceptibility to the CAC and its malignancy. Increased mucosal inflammation, defective epithelial homeostasis accompanied the increased CAC in Villin-Cld1-Tg mice. We further found significantly increased levels of protumorigenic M2 macrophages and $\beta$-CateninSer552 ( $\beta$-CatSer552) expression in the CAC in Cld-1Tg versus WT mice. Mechanistic studies identified the role of PI3K/Akt signaling in Cld-1 dependent activation of the $\beta$-CatSer552, which, in turn, was dependent on pro-inflammatory signals. Our studies identify a critical role of Cld-1 in promoting susceptibility to CAC. Importantly, these effects of deregulated Cld-1 were not associated with altered tight junction integrity, but on its non-canonical role in regulating Notch/PI3K/Wnt/ $\beta$-CatSer552 signaling. Overall, outcome from our current studies identifies Cld-1 as potential prognostic biomarker for IBD severity and CAC, and a novel therapeutic target.
\end{abstract}

Users may view, print, copy, and download text and data-mine the content in such documents, for the purposes of academic research, subject always to the full Conditions of use:http://www.nature.com/authors/editorial_policies/license.html\#terms

To whom correspondence should be addressed: Punita Dhawan, Departments of Biochemistry and Molecular Biology, University of Nebraska Medical Center, Omaha, NE-68022, USA, Tel: (402)-559-6587; Fax: (402)-559-6650, punita.dhawan@unmc.edu.

Conflict of Interest

The authors declare that they have no conflict of interest. 


\section{Keywords}

Colitis associated cancer; Wnt signaling; Claudin-1; CAC: $\beta$-Catenin; AKT; Notch; Epithelial cell homeostasis

\section{Introduction}

Inflammatory bowel disease (IBD), a chronic and relapsing disorder of the gastrointestinal tract, significantly increases the chances of developing colon cancer (colitis-associated cancer (CAC)). Understanding why IBD patients are susceptible to CAC, can have significant impact on the clinical management and quality of the patient life and survival (1).

A growing body of evidence suggests that the alterations in normal tight junctions function, associates with and/or exacerbates IBD (2). Claudins are now confirmed to be the integral component of TJ-structure/function (3). Among claudin proteins, claudin-1 has attained peculiar significance in promoting spontaneous colon cancer (CRC) (4-7). In our laboratory, using a mouse model engineered to mimic upregulated Cld-1 levels (Cld-1Tg mice), as in CRC patients, we have demonstrated that increased Cld-1 modulates intestinal epithelial homeostasis by hyperactivating Notch-signaling, and promotes DSS-induced colitis (6). A remarkable finding in our published study was that Cld-1Tg mice demonstrated persistent inflammation and impaired epithelial restitution despite hyper-proliferation (4). A proinflammatory milieu and hyper-proliferation can create perfect recipe for carcinogenesis and therefore we postulated deregulated Cld- 1 expression to be predictive of CAC risk in IBD patients, and to promote disease severity. Current study was undertaken to test this hypothesis as a role of Cld-1 in CAC remain untested.

Importantly, a key role of the canonical Wnt-mediated $\beta$-catenin activation in regulating intestinal stem cell niche and therefore injury/repair is well recognized (8). Accordingly, deregulated $\beta$-catenin signaling promotes colon cancer $(9,10)$. In colitis-associated regeneration and/or CAC, upregulated expression and nuclear accumulation of Ser-552 phosphorylated $\beta$-Catenin ( $\mathrm{p}-\beta-\mathrm{Cat}{ }^{\mathrm{S} 552}$ ) has been found to be of prime importance (11). A cross-talk between Cld-1 and $\beta$-Catenin has been reported previously by our lab and of others $(5,12,13)$. However, whether deregulated Cld-1 modulates the $\mathrm{p}-\beta-\mathrm{Cat}{ }^{\mathrm{S} 552}$ signaling for its effects upon colitis and associated neoplasia remains unclear.

In current study, we have carefully determined the role of deregulated Cld-1 expression in $\mathrm{CAC}$ and underlying cellular and molecular modalities using a well-constructed study scheme and mouse models of DSS colitis/recovery and CAC (AOM/DSS) (14). Based on these mechanistic investigations, we provide firm evidence that an upregulated Cld-1 in intestinal epithelial cells render mice susceptible to CAC. Deregulation of the intestinal epithelial cell homeostasis emerged as the principal contributor to this phenotype as Cld-1Tg mice failed to recover from colitis despite hyper-proliferative crypt and rather displayed dysplastic crypts. Notably, Cld-1Tg mice not only showed an increased colonic tumor burden when subjected to the CAC, but also more malignant disease as tumors in Cld-1Tg mice were appeared earlier as compared to the WT-littermates. Further investigation identified Notch/Akt-led modulation of the $\beta$-Catenin pathway by specific induction of the 
ser552 moiety as the key signaling altered by deregulated Cld-1 during CAC. Taken together, these novel findings not only emphasize a critical role of Cld-1 in regulating intestinal epithelial homeostasis and therefore cancer but also a broader role of barrierintegral proteins in regulating mucosal epithelial and immune homeostasis than previously considered. In conclusion, our findings emphasize novel therapeutic significance of Cld-1 in limiting colitis severity and progression to the colon cancer, and its prognostic utility.

\section{Results:}

\section{In Cld-1Tg mice, colitis associated epithelial regeneration/repair is impaired and leads to dysplasia:}

We have previously reported that Cld-1Tg mice demonstrate exacerbated colitis and impaired recovery (4). To test, whether impaired recovery in these mice are simply a delayed response or a fundamental defect in epithelial homeostasis, we subjected these mice to DSScolitis ( 7 days) followed by an extended period of recovery (10 days post-DSS administration (Fig. 1A). External parameters including body weight loss in DSS-treated mice confirmed susceptibility to colitis and severity in Cld-1Tg mice versus WT-littermates (Fig. 1A). As reported previously, Cld-1Tg mice demonstrated defective recovery postcolitis and continued to lose weight while wild-type mice recovered to their normal weight (Fig. 1A). In support of severe and persistent colitis in Cld-1Tg mice, colon appeared significantly shorter than the WT mice (Supplementary Figure 1A-B). The pathological scoring of the overall colonic injury comprising inflammation, depth of inflammation and cryptal damage, were significantly higher in Cld-1Tg mice than the WT mice which were subjected to DSS recovery protocol (Fig. 1B and Supplementary Figure 1C-D). Interestingly, the histology of Cld-1 $\mathrm{Tg}$ mice showed impaired recovery containing dysplastic crypts (>80\% of Cld-1 Tg mice) compared to regenerative crypts in WT DSS recovery mice (Fig. 1B and 1C). We further examined if such a defect is limited to the DSSinduced colitis and recovery. Therefore, Cld-1Tg mice were subjected to yet another murine model of colitis where hyperproliferation associates with the disease intensity. Mice were subjected to the Citrobacter rodentium induced colitis as described in the "materials and methods". The colonic thickness and injury scores were significantly high in Cld-1 Tg mice infected with Citrobacter rodentium than WT mice (Supplementary Figure 3A-B). Remarkably, Cld-1Tg mice demonstrated similar impairment of recovery in C. rodentium infected mice too (Supplementary Figure 3C), despite hyper-proliferation as evidenced by Ki67 staining (Supplementary Figure 3D) similar to that observed in the DSS recovery model in Cld-1 Tg mice (Supplementary Figure 5A-B). This suggested a fundamental role of Cld-1 expression in maintaining colonic epithelial homeostasis.

\section{Deregulated Cld-1 expression potentiates susceptibility to CAC:}

Above observations that increased Cld-1 expression in the intestinal epithelium, results in persistent inflammatory conditions and epithelial cell plasticity, led us to believe that Cld-1 expression may also promote susceptibility and/or severity of CAC. To examine, Cld-1Tg and WT mice were subjected to the widely utilized murine model of the CAC by subjecting to AOM/DSS exposure, as described in methods section (Fig. 2A). Associated changes in the body weight suggested severe disease conditions in Cld-1Tg mice as body weight loss in 
these mice was significantly higher than the WT-mice subjected to same treatment (Fig. 2A). Longitudinal colonoscopic evaluation further determined precautious colonic tumor development in these mice and an increased tumor burden than age and sex-matched WTlittermates (Fig. 2B). These findings were supported by the data at the time of the mice sacrifice, which demonstrated significant increases in colonic tumor burden, tumor size and colon weight (Fig. 2B). Histopathological analysis of Swiss-rolled colons by a blinded, experienced mouse gastrointestinal pathologist (K.W) confirmed endoscopic observations, indicating increased overall inflammation, depth of inflammation and crypt damage involved (Supplementary Figure 4A-C). Histo-pathological examination of the tumors further demonstrated that Cld-1Tg mice had 100\% tumor penetrance (high-grade dysplasia) compared to the $<30 \%$ high-grade dysplasia in the WT cohort (Fig. 2C). The overall injury score was significantly high in Cld-1 Tg mice compared to WT mice under AOM/DSS exposure (Fig. 2C). Collectively, these results indicated a critical role for Cld-1 in accelerating colitis-associated tumorigenesis.

\section{Cld-1 expression is upregulated in Colitis associated Cancer (CAC):}

Considering the fact that increased Cld-1 expression led to increased susceptibility to CAC and also promoted its severity, we further determined if Cld- 1 expression is also upregulated in CAC and its association with disease severity. To test, we used the samples from chronic inactive colitis, mild to moderate dysplastic lesions (38 samples), low, grade (81 samples) and high grade dysplasia (17 samples) and colon cancer samples (25 samples) (Fig. 3A). Intensity of anti-Cld-1 immunostaining was determined by a GI pathologist (CS) in blinded manner. Outcome suggested a significant increase in Cld-1 expression (membrane +cytoplasmic; 4-5 fold) which was also stage specific (progressive increase from inactive colitis to Low grade/High grade dysplasia) (Fig. 3A and Supplementary Figure 5C). Similar increase in Cld-1 expression was observed in Cld-1Tg mice in Colitis associated Cancer mouse model (AOM/DSS) compared to WT control mice as evidenced by immunohistochemistry and immunoblotting (Fig. 3B and C). Overall, these data suggested that Cld-1 may serve as prognostic marker for the susceptibility of neoplasia growth in IBD patients.

\section{Altered immune environment in Cld-1Tg tumors:}

Considering inflammation is the key driving force in CAC and persistent inflammatory milieu in Cld-1Tg mice, we further determined if tumor microenvironment in Cld-1Tg mice differ from the WT-mice. Herein, we primarily examined immune cell infiltrate in the tumors from WT and Cld-1 Tg mice primarily for the T-cell infiltrate (CD3) and macrophages (F-480) using immunohistochemistry. A marked increase in CD3 and F480 immune cells in Cld-1Tg tumors supported a pro-inflammatory milieu in Cld-1Tg mice tumors ( $\mathrm{p}<0.01$ ) (Fig. 4A and B). However, considering that the macrophages identified by F480 can be pro- or anti-tumorigenic, we performed further analysis using specific markers for the M1 macrophages (antitumoral, the nitric oxide synthase (iNOS)) and M2 macrophages (protumorigenic, arginase1(Arg1)) (28) respectively. It was interesting that the levels of F-480+/iNOS+ macrophages, indicative of M1 phenotype, was quite diminished while levels of the F-480+/Arg1+ macrophages, indicative of M2 phenotype, was markedly higher in Cld-1Tg tumors (Fig. 4B). Our additional data for DNA damage using pH2AX 
expression, which is known to increase with inflammation $(29,30)$ further supported a proinflammatory environment in Cld-1Tg mice tumors versus WT-mice (Fig. 4C). Collectively, these data suggested a pro-tumor inflammatory microenvironment in Cld-1Tg tumors.

\section{Deregulated proliferation/apoptosis ratio characterized Cld-1Tg mice tumors.}

Pending increased tumorigenesis in Cld-1Tg mice, we postulated modulation of the proliferation/apoptosis ratio in these mice when subjected to the CAC. To examine potential alteration in the dynamic proliferation in the colonic epithelium in AOM/DSS treated mice we injected BrdU before sacrificing them. Tissue samples were subjected to Ki67, anti-Brdu, and anti-cleaved-caspase-3 immuno-detection, which demonstrated that the cell proliferation was markedly increased in Cld-1 mice tumors (Fig. 5A and B). Conversely, staining for cleaved caspase-3 indicated decrease in intra-tumoral apoptosis in Cld-1Tg mice as compared to WT mice (Fig. 5C). Taken together, the normal balance between apoptosis and proliferation was skewed towards hyperproliferative milieu in Cld-1Tg mice compared to the WT-mice.

\section{Cld-1 mediated colonic epithelial plasticity and CAC progression is a product of over- activated $\beta$-Cat ${ }^{\text {ser552 }}$ signaling:}

The dysregulation of $\beta$-Catenin homeostasis is a key indicator of the hyperactive Wnt signaling (15), and $\beta$-Catenin is a mutational target in colitis-associated carcinogenesis, based on its increased expression and altered subcellular distribution (11). We have previously demonstrated a cross talk between Cld-1 and the Wnt-signaling (6). Therefore, we postulated that the increased Cld-1 levels in colitis may also perturb the Wnt-signaling to promote tumorigenesis. We therefore analyzed $\beta$-Catenin status by immunohistochemistry which indeed demonstrated an excessive cytoplasmic and nuclear $\beta$-Catenin localization in Cld-1Tg tumors compared to WT tumors (Fig. 6A). To further assess if Wnt-signaling is activated, we determined expression of Wnt-signaling targets including cyclin-D1, cancer stem cell markers such as CD133 and Aldh1 (Fig. 6B-C). Together, these findings suggest that the increased CAC in Cld-1Tg is positively associated with Wnt-signaling and expression of cancer stem cells (CSC) as compared to WT tumor tissues.

\section{Cld-1 regulates $\beta$-Catenin through its phosphorylation at Ser552 to promote CAC:}

Importantly, previous studies have observed nuclear accumulation of Ser552 phosphorylated $\beta$-Catenin ( $\mathrm{p}-\beta$-Cat ${ }^{\mathrm{Ser}-552}$ ) in intestinal epithelial cells (IEC-6) during colitis and colitisassociated cancer (16). To investigate the possible mechanism of Cld-1 mediated regulation over $\beta$-catenin, we focused on the transcriptional activation and nuclear accumulation of $\beta$ catenin by phosphorylation on residues Ser552 in CAC model. To examine this possibility colonic tissues from WT and Cld-1Tg mice subjected to AOM/DSS were stained for $\mathrm{p}-\beta$ $\mathrm{Cat}^{\mathrm{Ser}-552}$. The results show an increased nuclear accumulation of $\mathrm{p}-\beta-\mathrm{Cat}{ }^{\mathrm{Ser}-552}$ in the dysplastic region from DSS recovery and AOM/DSS induced animals compared to control animals (Fig. 7A). This accumulation was more intense in the tumors of Cld-1 Tg mice compared to WT tumors. Similar results were observed in the immunoblotting analysis of the colonic tissue lysates which proves a significant increase in the expression of $p-\beta$ $\mathrm{Cat}^{\mathrm{Ser}-552}$ in the Cld-1Tg mice subjected to AOM/DSS (Fig. 7B). Together, these data support the hypothesis that nuclear $\beta$-catenin accumulation is an important event which 
contributes to the deleterious effect of Cld-1 in establishing dysplasia and progressing tumorigenesis in CAC.

\section{Cld-1 regulates $\beta$-Catenin through Notch/PI3K/Akt pathway in colon cancer cells:}

To understand the mechanistic details of signaling involved, we used in vitro colon cancer cells, HT-29 ${ }^{\mathrm{C}}$, HT-29 ${ }^{\mathrm{Cld}-1}$ cells as well as normal intestinal epithelial IEC $^{\mathrm{C}}$ and IEC ${ }^{\mathrm{Cld}-1}$ cells. These cells were treated with TNF-a, an inflammatory cytokine to mimic in vivo conditions. Our immunoblotting analysis demonstrated an increase in nuclear $\beta$-Catenin accumulation in HT-29 ${ }^{\mathrm{Cld}-1}$ and IEC ${ }^{\text {Cld-1 }}$ cells compared to HT-29 $/$ IEC $^{\mathrm{C}}$ cells. TNF-a treatment further led to significant accumulation of nuclear $\beta$-Catenin in HT-29Cld-1 and IEC ${ }^{\text {Cld- } 1}$ cells (Fig. $8 \mathrm{~A}$ and Supplementary Fig 6A). Previous studies have shown PI3K/Akt pathway and PKA pathway can phosphorylate $\beta$-Catenin at Ser552. Furthermore, we have shown in our previous studies that Cld-1Tg mice demonstrate Notch activation (4) and has been demonstrated a cross talk between Notch and PI3K/Akt pathway (17). Thus, we hypothesized Notch, PI3k/Akt and or PKA pathway might play an important role in Cld-1 mediated phosphorylation of $\beta$-Catenin. To test, we examined the activation of Notch, PI3K/Akt and PKA (p-CREB) in Cld-1 overexpressing cells under above conditions. Overexpression of Cld-1 induced Cleaved Notch, p-Akt and p-CREB activation which was further induced on treatment with TNF- $a$ in Cld-1 overexpressing HT-29 cells (Fig. 8A). Similar increase in HES-1 and p-Akt expression was observed in tumors from Cld-1Tg mice subjected to AOM/DSS model (Supplementary Fig 6E-F). To correlate it with functional phenotype, we determined the proliferation in these cells. The rate of proliferation was significantly more in HT-29 Cld-1 cells compared to HT-29C cells which was further increased by TNF- $a$ treatment. This increase in cell proliferation upon TNF- $\alpha$ treatment was more pronounced in HT-29 ${ }^{\mathrm{Cld}-1}$ cells than HT-29 $\mathrm{C}$ cells which proves that Cld-1 mediated increase in proliferation (Fig. 8C).

Next we determined whether inhibiting Notch could suppress TNF-a/Cld-1-induced $\beta$ Catenin phosphorylation (S552) in HT-29 Cld-1 cells. Cells were pretreated with or without DAPT $(50$ and $100 \mu \mathrm{M})$ for 1 hour, and then stimulated with TNF- $\alpha(10 \mathrm{ng} / \mathrm{mL})$ at the indicated times. As shown in Fig. 8B treatment of cells HT-29 ${ }^{\mathrm{Cld}-1}$ with TNF-a enhanced phosphorylation of $\beta$-Catenin at ser552 (which closely correlates with nuclear accumulation), and was inhibited by pretreatment with DAPT (Notch inhibitor). Most interestingly, inhibition of Notch signaling inhibited p-Akt activation while did not have any effect on p-CREB activation suggesting that Cld-1 mediated Notch activation induces p-Akt to phosphorylate and activate $\beta$-Catenin. To further confirm these findings, we used LY294002, a specific inhibitor of PI3K/Akt pathway. Cells were pretreated with 10 and 20 $\mu \mathrm{M}$ LY294002 for 1 hour and then exposed to TNF-a. Inhibition by LY294002 inhibited pAkt and $\mathrm{p}-\beta-\mathrm{Cat}{ }^{\mathrm{Ser}-552}$ suggesting again role of Akt activation downstream of Cld- 1 and Notch to activate $\beta$-Catenin signaling (Fig. 8B). The increased rate of proliferation in HT-29 ${ }^{\text {Cld }-1}$ cells upon TNF- $\alpha$ treatment was significantly inhibited by DAPT inhibitor proving the role in Notch/Akt/p- $\beta$-catenin signaling in Cld- 1 mediated proliferation of cancer cells (Fig. 8B). Combined together, Cld- 1 modulates $\beta$-Catenin phosphorylation and activation by regulating Notch/Akt signaling to regulate colitis associated cancer (Supplementary figure 7). 


\section{Discussion:}

A constant increase in the incidence of inflammatory bowel disease (IBD) and therefore susceptibility to, and incidence of, colitis-associated cancer, requires an improved understanding of the molecular modalities, for therapeutic gains (1). It is here worthy of noting that despite many similarities in the disease characteristics including the cancer promoting role of pro-inflammatory signaling, the genetic and molecular regulatory mechanisms of the CRC and CAC are different (18). In fact, recent studies have provided increasing evidence for contrasting effects of specific genes, related specifically with proliferative and migratory abilities of the IECs, upon CAC and CRC pathogenesis $(19,20)$. In this regard, mucosal healing involving increased proliferation and cell mobility helps lessen IBD severity and thus CAC whereas these mechanisms promote CRC $(21,22)$. Thus, considering the known role of deregulated Cld-1 expression in modulating colonic epithelial homeostasis and the CRC, and the fact that Cld-1 expression is also altered in IBD, it was imperative that we determine whether deregulated Cld-1 expression in IBD is good or bad in relation to the susceptibility to CAC and disease severity (4). The outcome from current study now clearly establish that deregulated Cld-1 expression in IBD not only helps promote susceptibility to the CAC but also its malignancy, as in the case of the CRC. Yet other noteworthy outcomes in current study are that: 1) Cld-1 deregulation promotes IBD and CAC susceptibility and severity by not destabilizing the tight junction integrity and permeability, as would be the conventional explanation, but by deregulating IEC homeostasis (proliferation and cell fate determination) by modulating key signaling events implicated in regulating the IEC niche; and 2) claudin-1 modulates both, CRC and CAC, by modulating the $\mathrm{Wnt} / \beta$-catenin signaling however underlying regulatory mechanisms differ markedly. Overall, the outcome from this comprehensive analysis identifies prognostic significance of Cld-1 in susceptibility to the CAC among IBD patients and a novel therapeutic target.

Importantly, the notion that chronic inflammation is the driving force for the CAC development, and hence increased susceptibility amongst IBD patients is widely accepted $(20,23)$. Our previous report that mice with constitutive increase in the expression of Cld-1 in their colonic epithelium, to model pathological Cld-1 levels in IBD-patients, demonstrate persistent inflammation when subjected to experimental colitis syncs well with our current findings that Cld-1 Tg mice have increased susceptibility to the CAC and disease severity (4). However, noteworthy feature of our previous report was that upregulated colonic Cld-1 expression was not associated primarily with the barrier permeability defects, as would be expected, and rather indirectly due to the unexpected modulation of the colonic epithelial homeostasis and goblet cell differentiation, and hence Muc-2 expression (4). Our findings that Cld-1 modulated Notch-signaling in the colonic epithelium by increasing ligand release in MMP-2/9-dependent manner then provided the first glimpse in a novel however noncanonical role of Cld-1 in the maintenance of the stem cell niche and cell fate determination (4). Taken together, our previous studies suggested that an increased susceptibility to colitis and persistent disease even during the recovery period (when mice are transferred to regular water after DSS-colitis for 7-days) in Cld-1Tg mice were byproduct of altered colonic epithelial homeostasis (4). A role for the Notch-signaling in regulating colonic epithelial cell homeostasis and injury/repair is now well recognized. 
However, a key question that begged further explanation was that whether increased colitis severity in Cld-1Tg mice was simply a reflection of a delayed recovery. The extended recovery study where WT and Cld-1Tg mice were allowed to recover from acute DSS-colitis $(2.5 \% \mathrm{~W} / \mathrm{V}$ in drinking water) for 10 days now clearly demonstrate that such is not the case, and Cld-1Tg mice have an inherent disability in repairing the colonic epithelia post-colitis despite an increased propensity of proliferation. As described, we unexpectedly found dysplastic appearance in the colon in Cld-1Tg mice that were subjected to DSS-colitis and recovery compared to the WT-mice which demonstrated expected recovery and return to the normal body weight. In this regard, wound healing consists of an inflammatory phase followed by epithelial cell proliferation and tissue remodeling. Tissue injury induces immediate recruitment of neutrophils and infiltrating leukocytes. This is followed by proliferative phase of wound healing (24). However, a defect in the ability of stem cells to differentiate appropriately into the proper colonic epithelial cell lineage could result in impaired wound healing despite the expansion of the proliferative cell ratio (25). We suspect such is the case in our study model and persistent inflammation and dysplastic epithelium is an output of failure to properly differentiate by stem cells in Cld-1Tg mice. However, DSScolitis is a complex model to clearly dissect these cellular events in their totality and we are currently implying other models of stem cell injury, focusing primarily on inhibiting Notchsignaling, for better understanding of these possibilities. Taken together, above findings highlight the clinical significance of deregulated Cld-1 levels in IBD patients as we postulate that Cld-1 expression analysis can be developed into a prognostic biomarker for predicting susceptibility to the severe disease and colon cancer amongst ulcerative colitis patients.

The fact that the deregulated Cld-1 expression modifies the homeostasis of the colitisassociated epithelial injury/repair is further supported by our findings that Wnt/ $\beta$-catenin signaling and target gene expression. It was however interesting that it was $\beta$-Catenin ser552-phosphorylation that was impacted in Cld-1Tg mice when subjected to colitis. Of note, recent studies have highlighted the essential role of this signaling mechanism in colitisassociated injury/repair and colon carcinogenesis $(11,26)$. Our findings that the Wnt/ $\beta$ Catenin ${ }^{\mathrm{Ser} 552}$ signaling was upregulated even in colon cancer cells overexpressing Cld-1 suggests an epithelial cell intrinsic effect of the upregulated Cld-1 levels upon Wnt/ $\beta$-catenin signaling. Such an inference is well supported by previous findings from our laboratory and of others $(26,27)$. A sharp increase in the expression of various stem cell markers that are also considered to label cancer stem cells further supports our postulation that deregulated Cld-1 expression modifies signaling cascades, possibly through the tight regulation of the notch- and Wnt-signaling, for creating an imbalanced stem cell proliferation/differentiation program and thus impaired injury/repair despite an increased propensity of cell proliferation. We are currently in the process of further delineating the interactions between the Notchand Wnt-signaling impacted by Cld-1 expression for its observed effects on colitis and CAC.

However, our data suggest that the deregulation of colonic epithelial homeostasis integrates with the pro-inflammatory mechanisms for the deleterious effects of Cld- 1 on colitis and CAC. Our data from cultured cells where exogenous administration of tumor necrosis factor (TNF-a) further potentiates the expression of the Wnt/ $\beta$-Cat ${ }^{\mathrm{Ser} 552}$ supports such a postulation. Moreover, we have found that the tumor-associated macrophages (TAMs) the M2 phenotype is significantly enriched in the tumors in Cld-1Tg mice subjected to the CAC 
than the WT mice. A role for the TAMs and tumor-associated dendritic cells (TADC) as the source of pro-inflammatory mediators in the tumor microenvironment is now well recognized $(28,29)$. However, we have also documented a significant increase in the T-cell infiltration in Cld-1Tg mice tumors. Thus, further studies aimed at careful dissection of the T-cell population being enriched in Cld-1Tg mice during colitis versus macrophage population can be of significant information, and part of our future studies. In this regard, a growing body of evidence now supports an important role of the deregulated epithelial junctional constituents in promoting mucosal inflammation and colitis-associated cancer. For example, mice expressing a dominant negative $\mathrm{N}$-cadherin in colonic epithelium develop spontaneous mucosal inflammation and colitis-associated dysplasia, and significant alterations in the proliferative/differentiating balance of colonic epithelial cells (30). Similarly, Junctional adhesion molecule (Jam-A) knock out results in a dramatic increase in susceptibility to DSS-induced colitis. Deletion of Bves, a tight junction-associated protein, renders similar effects and augments inflammatory carcinogenesis (31). Also, we have recently demonstrated that loss of Cld-3 (Cld-3-/- mice) led to a significant increase in $\mathrm{CAC}$ and its malignancy, however, in these mice we also noticed a significant impairment of the mucosal barrier function (32). Taken together, studies including our current study now establish the mucosal barrier proteins as critical regulators of the intestinal epithelial and immune homeostasis. The diverse mechanisms these proteins may affect colitis severity and susceptibility the CAC not only highlight the complexity and dynamisms of the mucosal barrier in intestinal homeostasis but also entails specific prognostic/therapeutic potential of these proteins for improving the clinical management and patient survival.

Our additional studies identified Notch/Akt activation as the signaling moiety mediating the effects of deregulated Cld-1 upon Wnt/ $\beta$-Cat ${ }^{S e r 552}$ signaling. Notably, we have now confirmed an upregulated and deregulated Cld-1 expression in CAC patient specimen while previous studies have revealed that the human colitis tissue have increased expression of the nuclear $\mathrm{p}-\beta-\mathrm{Cat}^{\mathrm{Ser}-552}$ (16). Of interest, expression of PTEN, a tumor suppressor, is negatively regulated by HES-1, a Notch signaling target $(33,34)$. PTEN deficiency increases PI3K/Akt activation which has been shown to increase $\mathrm{p}-\beta-\mathrm{Cat}^{\mathrm{Ser}-552}$ expression, proliferation and crypt fission (35). Thus, we predict that the deregulation of Cld-1 expression in the colon modulates Wnt/ $\beta$-Cat ${ }^{\text {Ser552 }}$ signaling in Notch/ PI3K/Akt-dependent manner. Notably, we have previously reported Akt-dependent Wnt-signaling upregulation by overexpressing Cld-1 in colon cancer cells (36). Notably, in melanoma, Notch activation synergizes with Wnt signaling to promote tumorigenic ability of the primary tumor cells, but it does not activate Wnt signaling in normal melanocytes $(37,38)$. Thus, cross-talk between Notch and Wnt/ $\beta$-Catenin signaling under tumorigenic environment in promoting CAC in Cld-1Tg mice is highly plausible. In this regard, we have previously demonstrated that offspring mice from the cross between $\mathrm{APC}^{\mathrm{min}}$ mice and $\mathrm{Cld}-1 \mathrm{Tg}$ mice demonstrate a significant upregulation in the colon tumor burden and tumor malignancy compared to the $\mathrm{APC}^{\mathrm{min}}$ mice when subjected to the DSS-colitis (6). Combined together, our results suggest that Cld-1 regulates $\beta$-Catenin activation through Notch and AKT dependent manner to promote $\mathrm{CAC}$, and thus identifies Cld-1 as potential biomarker for susceptibility to the ulcerative colitis and CAC, and a novel therapeutic target albeit further studies are needed to clearly define similarities and differences in the molecular undertakings of the effects of 
deregulated Cld-1 expression in promoting CRC and CAC, and part of our ongoing investigations.

\section{Materials and Methods}

Animals:

Cld-1 Tg mice were generated as described previously (4). Briefly, human Cld-1 cDNA were cloned into pBS plasmid vector under the control of the intestine specific villin promoter. The founder lines were of C57BL/6 genetic background and offspring genotyped using DNA isolated from the tail genomic DNA. Presence of the transgene was verified by PCR using two different PCR primer sets. All mice had access to water and to the same standard rodent diet ad libitum Animal studies were carried out in accordance with the approved protocols by institutional animal ethics committee (IACUC) of UNMC on Use and Care of Animals.

\section{Induction of colitis/recovery and AOM/DSS mouse model:}

The induction of colitis was carried out using the standard protocol (39). Mice (8-10 weeks old) $(\mathrm{n}=8)$ received $2.5 \%$ dextran sulfate sodium (TdB consultancy AB, Sweden) in sterile drinking water (colitis) for 7 days and replaced with sterile drinking water after $7^{\text {th }}$ day and continued until the end of the experiment (DSS recovery). The Citrobacter rodentium strain DBS100 (ATCC 51459; American Type Culture Collection) was grown by shaking overnight in Luria-Bertani broth at $37^{\circ} \mathrm{C}$. Cld- $1 \mathrm{Tg}$ mice and WT mice were orally inoculated with $2 \times 10^{9} \mathrm{CFU}$ C. rodentium in a total volume of $200 \mu \mathrm{l}$ per mouse. AOM application was performed by standard protocol (40). For AOM/DSS model, mice (8-10 weeks old) $(\mathrm{n}=8)$ received $10 \mathrm{mg}$ per kg body weight AOM (Sigma Chemical Co, St. Louis, MO, USA) per intraperitoneal injection. Following the single AOM injection, mice were treated with $2.5 \%$ DSS in drinking water from days 1 to $5 ; 22$ to 26 and 43 to 47 respectively.

\section{Histology:}

The colon was excised, rinsed with PBS (pH 7.4) to remove fecal material, placed on a piece of filter paper, opened longitudinally, laid open and fixed. For histological analysis, colonic swiss rolls were fixed in $4 \%$ neutral buffered formalin overnight, processed through paraffin, sectioned at $5 \mu \mathrm{m}$, and stained with $\mathrm{H} \& \mathrm{E}$. Histological assessment was performed by a pathologist (K.W) who was blinded to the experimental protocol Colitis scores were calculated based on a graded scale of inflammation described previously(41). Combined colitis scores are the sum of the scores. For tumor histology, colon tumors sized $\geq 2 \mathrm{~mm}$ in diameter were resected, including adjacent normal tissue, dehydrated and embedded in paraffin and 4- $\mu \mathrm{m}$ tissue sections cut in parallel with the mucosal surface and stained with H\&E. Histopathologic analysis of neoplastic lesions was performed using standard criteria according to the classification of human adenomas of the colon and the assessment of the degree of dysplasia. 


\section{Immunohistochemistry:}

Colon tissues of $5 \mu \mathrm{m}$ thickness were deparaffinised in histoclear and rehydrated using graded series of ethanol solutions $(100 \%, 90 \%, 70 \%, 50 \%, 30 \%)$. The slides were incubated in antigen retrieval buffer ( $\mathrm{pH} 6.0$ ) at $95-100{ }^{\circ} \mathrm{C}$ for 10 minutes each in a programmed pressure cooker. The sections were then allowed to cool at room temperature and then rinsed with $1 \mathrm{x}$ Tris buffered saline (TBS), and the endogenous peroxidase activity was quenched using $0.3 \% \mathrm{H}_{2} \mathrm{O}_{2}$ in methanol for $30 \mathrm{~min}$. The sections were rinsed with Tris buffered saline (TBS). Sections were incubated with respective antibodies followed by the VECTASTAIN ABC kit (Vector Laboratories, Burlingame, CA). Sections were developed using 3,3 ${ }^{1}$ diaminobenzidine tetrahydrochloride (DAB) chromogen. The sections were counterstained using hematoxylin, dehydrated (70\%, 90\% and $100 \%)$, mounted and visualized under the light microscope. Quantitative analysis was performed by averaging positive cells across 20 randomly selected fields.

\section{Immunoblotting:}

Protein lysates from mouse tissue samples/ cells were prepared using lysis buffer $(20 \mathrm{mM}$ Tris- $\mathrm{HCl}, 200 \mathrm{mM} \mathrm{NaCl}, 0.2 \%$ Nonidet P-40, $0.5 \%$ Triton X-100 and protease inhibitors) and boiled at $100^{\circ} \mathrm{C}$ for $10 \mathrm{~min}$. Equal amounts of protein extracts were separated on $10 \%$ polyacrylamide gels by using standard sodium dodecyl sulfate-polyacrylamide gel electrophoresis (SDS-PAGE), then transferred onto PVDF membranes (Biorad). Following blocking in Tris-buffered saline (TBS) with $0.1 \%$ Triton X-100 and 5\% milk, the membranes were immunodetected using following antibodies, $\beta$-Catenin; $\mathrm{p}-\beta$-Cat ${ }^{\mathrm{Ser} 552}$, Total-AKT, $\mathrm{p}$ AKT, Cleaved notch, Cleaved caspase-3, Cyclin-D1, HES-1, iNOS, F-480, Claudin-1 and $\beta$ Actin (Cell Signaling Technology, Inc. Danvers, MA).

\section{HT-29/IEC-6 Cell transfection:}

HT-29 and IEC-6 cells were cultured in RPMI-1640 medium (Hyclone, Logan, UT, USA) supplemented with $10 \%$ fetal bovine serum and $1 \%$ antibiotics (penicillin and streptomycin). The cells were incubated at $37^{\circ} \mathrm{C}$ in a $5 \% \mathrm{CO}_{2}$ atmosphere. HT-29/IEC cells were plated in $10-\mathrm{cm}$ dishes at $60 \%$ confluence 1 day before transfection. Cells were transfected with pCMV plasmids encoded Claudin-1 using the jet PRIME transfection reagent (PolyplusTransfection, New York, NY), $48 \mathrm{~h}$ after transfection, cells were harvested and used for analysis. Maintained under selection pressure with $200 \mu \mathrm{g} / \mathrm{ml} \mathrm{G} 418$. For experiments to study the effect of TNF-a (R\&D Systems Inc, Minneapolis, MN), the HT-29/IEC control or Cld-1 overexpressed cells were exposed to TNF- $a$ and incubated for different time durations of $5 \mathrm{~min}, 30 \mathrm{~min}, 1$ hour, 8 hours, 12 hours and 24 hours. Assays were repeated three times for each condition.

\section{DAPT and LY294002 treatment:}

HT-29/IEC-6 control and Cld-1 overexpressed cells were treated by DAPT (50 and $100 \mu \mathrm{M}$ ) or LY294002 (10 and $20 \mu \mathrm{M})$ (Calbiochem Inc., USA) for 24 hours, respectively. After treatments, the proteins were extracted and immunodetected by the required antibodies. The cell proliferation was detected by presto blue cell viability reagent (Thermo fisher scientific) assay. 


\section{Human specimens:}

Tissue microarrays (TMAs) were made from paraffin embedded tissue blocks of colectomy specimens from patients with inflammatory bowel disease. The collection of human tissue was approved by Vanderbilt Institutional Research Board. The TMAs used in the current study contains specimens from normal (14), CIC (27), moderate (38), low grade dysplasia (81), high grade dysplasia (17) and colon cancer patient colon sections (25). These sections were stained with Cld- 1 antibody to detect the comparative analysis of Cld-1 and subcellular localization during the step wise progression to colon cancer in humans suffering from IBD.

\section{Statistical Analysis:}

The obtained data's were analyzed using SPSS/10.0 software. Hypothesis testing methods included one-way analysis of variance (ANOVA) followed by post hoc Tukey's test. All these results were expressed as Mean \pm S.D for six animals in each group and $p$ values of less than $0.05(\mathrm{p}<0.05)$ were considered as statistically significant.

\section{Supplementary Material}

Refer to Web version on PubMed Central for supplementary material.

\section{Acknowledgements}

This study was supported by BX002086 (VA merit), CA216746 (NIH/NCI) and a pilot project award from Fred and Pamela Buffet Cancer Center, which is funded by a National Cancer Institute Cancer Center Support Grant under award number P30 CA036727 to P.D and DK088902 (NIH/NIDDK) and BX002761 (VA merit) to A.B.S.

\section{References:}

1. Sengupta N, Yee E, Feuerstein JD. Colorectal Cancer Screening in Inflammatory Bowel Disease. Dig Dis Sci 2016;61(4):980-9. [PubMed: 26646250]

2. Landy J, Ronde E, English N, Clark SK, Hart AL, Knight SC, et al. Tight junctions in inflammatory bowel diseases and inflammatory bowel disease associated colorectal cancer. World J Gastroenterol 2016;22(11):3117-26. [PubMed: 27003989]

3. Gunzel D, Yu AS. Claudins and the modulation of tight junction permeability. Physiol Rev 2013;93(2):525-69. [PubMed: 23589827]

4. Pope JL, Bhat AA, Sharma A, Ahmad R, Krishnan M, Washington MK, et al. Claudin-1 regulates intestinal epithelial homeostasis through the modulation of Notch-signalling. Gut 2014;63(4):62234. [PubMed: 23766441]

5. Dhawan P, Singh AB, Deane NG, No Y, Shiou SR, Schmidt C, et al. Claudin-1 regulates cellular transformation and metastatic behavior in colon cancer. J Clin Invest 2005;115(7):1765-76. [PubMed: 15965503]

6. Pope JL, Ahmad R, Bhat AA, Washington MK, Singh AB, Dhawan P. Claudin-1 overexpression in intestinal epithelial cells enhances susceptibility to adenamatous polyposis coli-mediated colon tumorigenesis. Mol Cancer 2014;13:167. [PubMed: 24997475]

7. Ouban A Claudin-1 role in colon cancer: An update and a review. Histol Histopathol 2018:11980.

8. Liu L, Rao JN, Zou T, Xiao L, Smith A, Zhuang R, et al. Activation of Wnt3a signaling stimulates intestinal epithelial repair by promoting c-Myc-regulated gene expression. Am J Physiol Cell Physiol 2012;302(1):C277-85. [PubMed: 21975427]

9. Deitrick J, Pruitt WM. Wnt/beta Catenin-Mediated Signaling Commonly Altered in Colorectal Cancer. Prog Mol Biol Transl Sci 2016;144:49-68. [PubMed: 27865466] 
10. Sabatino L, Pancione M, Votino C, Colangelo T, Lupo A, Novellino E, et al. Emerging role of the beta-catenin-PPARgamma axis in the pathogenesis of colorectal cancer. World J Gastroenterol 2014;20(23):7137-51. [PubMed: 24966585]

11. Goretsky T, Bradford EM, Ryu H, Tahir M, Moyer MP, Gao T, et al. A Cytosolic Multiprotein Complex Containing p85alpha Is Required for beta-Catenin Activation in Colitis and Colitisassociated Cancer. J Biol Chem 2016;291(8):4166-77. [PubMed: 26565021]

12. Miwa N, Furuse M, Tsukita S, Niikawa N, Nakamura Y, Furukawa Y. Involvement of claudin-1 in the beta-catenin/Tcf signaling pathway and its frequent upregulation in human colorectal cancers. Oncology research 2001;12(11-12):469-76. [PubMed: 11939410]

13. Shiou S-R, Singh AB, Moorthy K, Datta PK, Washington MK, Beauchamp RD, et al. Smad4 Regulates Claudin-1 Expression in a Transforming Growth Factor- $\beta$-Independent Manner in Colon Cancer Cells. Cancer Research 2007;67(4):1571-9. [PubMed: 17308096]

14. Clapper ML, Cooper HS, Chang WC. Dextran sulfate sodium-induced colitis-associated neoplasia: a promising model for the development of chemopreventive interventions. Acta Pharmacol Sin 2007;28(9):1450-9. [PubMed: 17723178]

15. Sebio A, Kahn M, Lenz HJ. The potential of targeting Wnt/beta-catenin in colon cancer. Expert Opin Ther Targets 2014;18(6):611-5. [PubMed: 24702624]

16. Lee G, Goretsky T, Managlia E, Dirisina R, Singh AP, Brown JB, et al. Phosphoinositide 3-kinase signaling mediates beta-catenin activation in intestinal epithelial stem and progenitor cells in colitis. Gastroenterology 2010;139(3):869-81, 81 e1-9. [PubMed: 20580720]

17. Villegas SN, Gombos R, Garcia-Lopez L, Gutierrez-Perez I, Garcia-Castillo J, Vallejo DM, et al. PI3K/Akt Cooperates with Oncogenic Notch by Inducing Nitric Oxide-Dependent Inflammation. Cell Rep 2018;22(10):2541-9. [PubMed: 29514083]

18. Breynaert C, Vermeire S, Rutgeerts P, Van Assche G. Dysplasia and colorectal cancer in inflammatory bowel disease: a result of inflammation or an intrinsic risk? Acta Gastroenterol Belg 2008;71(4):367-72. [PubMed: 19317276]

19. Low D, Mino-Kenudson M, Mizoguchi E. Recent advancement in understanding colitis-associated tumorigenesis. Inflamm Bowel Dis 2014;20(11):2115-23. [PubMed: 25337866]

20. Terzic J, Grivennikov S, Karin E, Karin M. Inflammation and colon cancer. Gastroenterology 2010;138(6):2101-14 e5. [PubMed: 20420949]

21. Dave M, Loftus EV Jr. Mucosal healing in inflammatory bowel disease-a true paradigm of success? Gastroenterol Hepatol (N Y) 2012;8(1):29-38. [PubMed: 22347830]

22. Ullman TA, Itzkowitz SH. Intestinal inflammation and cancer. Gastroenterology 2011;140(6): 1807-16. [PubMed: 21530747]

23. Stidham RW, Higgins PDR. Colorectal Cancer in Inflammatory Bowel Disease. Clin Colon Rectal Surg 2018;31(3):168-78. [PubMed: 29720903]

24. Gonzalez AC, Costa TF, Andrade ZA, Medrado AR. Wound healing - A literature review. An Bras Dermatol 2016;91(5):614-20. [PubMed: 27828635]

25. Neal MD, Richardson WM, Sodhi CP, Russo A, Hackam DJ. Intestinal stem cells and their roles during mucosal injury and repair. J Surg Res 2011;167(1):1-8. [PubMed: 20599211]

26. Ormanns S, Neumann J, Horst D, Kirchner T, Jung A. WNT signaling and distant metastasis in colon cancer through transcriptional activity of nuclear beta-Catenin depend on active PI3K signaling. Oncotarget 2014;5(10):2999-3011. [PubMed: 24930890]

27. Brown JB, Cheresh P, Goretsky T, Managlia E, Grimm GR, Ryu H, et al. Epithelial phosphatidylinositol-3-kinase signaling is required for beta-catenin activation and host defense against Citrobacter rodentium infection. Infect Immun 2011;79(5):1863-72. [PubMed: 21343355]

28. Liu Y, Cao X. The origin and function of tumor-associated macrophages. Cell Mol Immunol 2015;12(1):1-4. [PubMed: 25220733]

29. Waniczek D, Lorenc Z, Snietura M, Wesecki M, Kopec A, Muc-Wierzgon M. Tumor-Associated Macrophages and Regulatory T Cells Infiltration and the Clinical Outcome in Colorectal Cancer. Arch Immunol Ther Exp (Warsz) 2017;65(5):445-54. [PubMed: 28343267]

30. Hermiston ML, Gordon JI. Inflammatory bowel disease and adenomas in mice expressing a dominant negative N-cadherin. Science 1995;270(5239):1203-7. [PubMed: 7502046] 
31. Parang B, Kaz AM, Barrett CW, Short SP, Ning W, Keating CE, et al. BVES regulates c-Myc stability via PP2A and suppresses colitis-induced tumourigenesis. Gut 2017;66(5):852-62. [PubMed: 28389570]

32. Ahmad R, Kumar B, Chen Z, Chen X, Muller D, Lele SM, et al. Loss of claudin-3 expression induces IL6/gp130/Stat3 signaling to promote colon cancer malignancy by hyperactivating Wnt/ beta-catenin signaling. Oncogene 2017;36(47):6592-604. [PubMed: 28783170]

33. Mendes RD, Canté-Barrett K, Pieters R, Meijerink JPP. The relevance of PTEN-AKT in relation to NOTCH1-directed treatment strategies in T-cell acute lymphoblastic leukemia. Haematologica 2016;101(9):1010-7. [PubMed: 27582570]

34. Wong GW, Knowles GC, Mak TW, Ferrando AA, Zúñiga-Pflücker JC. HES1 opposes a PTENdependent check on survival, differentiation, and proliferation of TCR $\beta$-selected mouse thymocytes. Blood 2012;120(7):1439-48. [PubMed: 22649105]

35. He XC, Yin T, Grindley JC, Tian Q, Sato T, Tao WA, et al. PTEN-deficient intestinal stem cells initiate intestinal polyposis. Nature genetics 2007;39(2):189-98. [PubMed: 17237784]

36. Singh A, Sharma A, Smith J, Krishnan M, Chen X, Eschrich S, et al. Claudin-1 up-regulates the repressor ZEB-1 to inhibit E-cadherin expression in colon cancer cells. Gastroenterology 2011;141:2140-53. [PubMed: 21878201]

37. Balint K, Xiao M, Pinnix CC, Soma A, Veres I, Juhasz I, et al. Activation of Notch1 signaling is required for $\beta$-catenin-mediated human primary melanoma progression. Journal of Clinical Investigation 2005;115(11):3166-76. [PubMed: 16239965]

38. Leong KG, Niessen K, Kulic I, Raouf A, Eaves C, Pollet I, et al. Jagged1-mediated Notch activation induces epithelial-to-mesenchymal transition through Slug-induced repression of Ecadherin. The Journal of Experimental Medicine 2007;204(12):2935-48. [PubMed: 17984306]

39. Chassaing B, Aitken JD, Malleshappa M, Vijay-Kumar M. Dextran sulfate sodium (DSS)-induced colitis in mice. Curr Protoc Immunol 2014;104:Unit 1525.

40. Snider AJ, Bialkowska AB, Ghaleb AM, Yang VW, Obeid LM, Hannun YA. Murine Model for Colitis-Associated Cancer of the Colon. Methods Mol Biol 2016;1438:245-54. [PubMed: 27150094]

41. Erben U, Loddenkemper C, Doerfel K, Spieckermann S, Haller D, Heimesaat MM, et al. A guide to histomorphological evaluation of intestinal inflammation in mouse models. Int J Clin Exp Pathol 2014;7(8):4557-76. [PubMed: 25197329] 

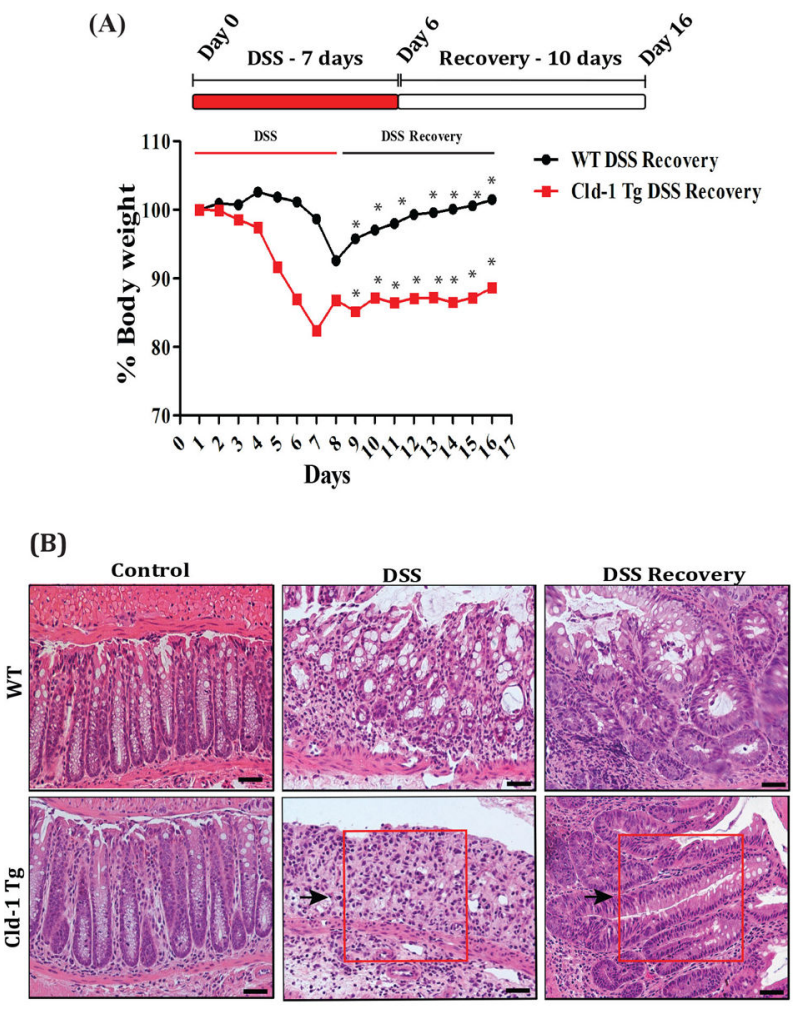

(C)
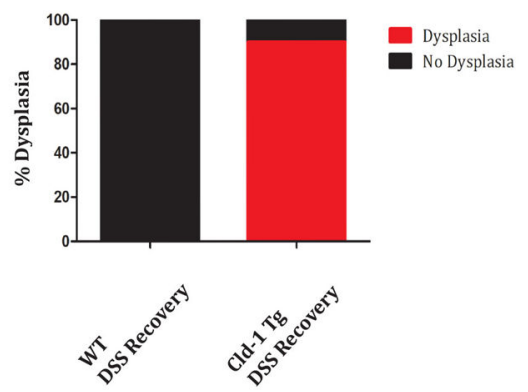

Fig. 1. Cld-1 intensifies colitis, leads to impaired recovery from DSS induction and triggers dysplasia:

(A) Schematic illustration of the experimental protocol. Age and sex matched WT ( $\mathrm{n}=8)$ and Cld-1 Tg (n=8) mice were treated with $2.5 \%$ DSS in drinking water for 7 days ad libitum (colitis group), followed by drinking water for 10 days (DSS recovery group). (B) The mean changes in body weight of the WT and Cld-1 Tg mice after being fed with 2.5\% DSS were measured every day until day 7 for colitis group and day 10 for DSS recovery group. (C) The percentage of mice possessing dysplastic crypt in the recovery protocol among WT and Cld-1 Tg groups. Results are statistically significant at $p<0.05$. Values sharing following symbols differ significantly, * - compared with WT DSS Recovery. 
(A)
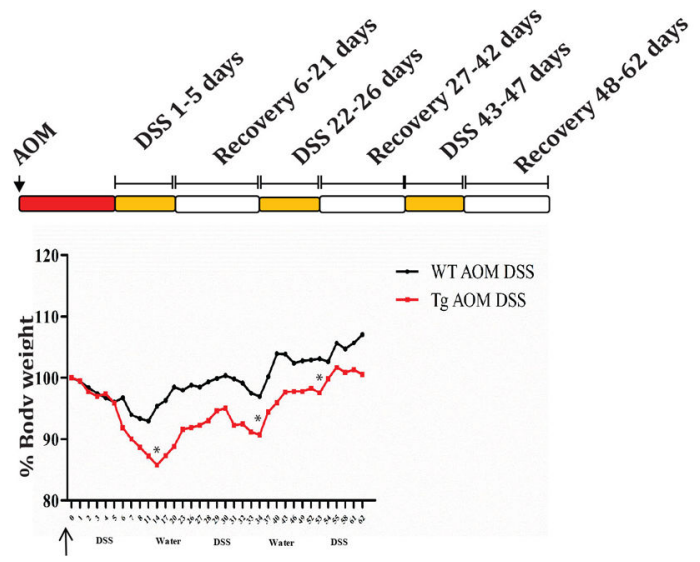

(B)
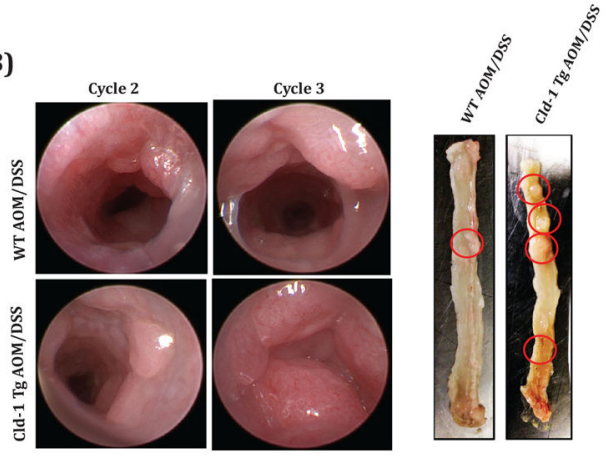

(C)
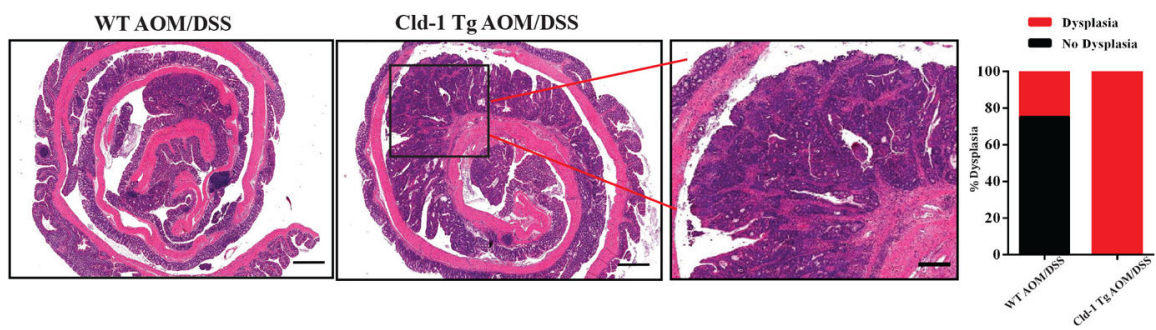

Fig. 2. Cld-1 Tg mice exhibited increased tumor burden following AOM/DSS exposure:

(A) Schematic overview of the AOM/DSS-induced colitis-associated colon cancer model, WT $(\mathrm{n}=8)$ and Cld-1 Tg mice $(\mathrm{n}=6)$ were intraperitoneally injected with AOM $(10 \mathrm{mg} / \mathrm{kg}$ body weight) and challenged with 3 cycles of DSS treatment (1 cycle representing 7 days of $2.5 \%$ DSS followed by 14 days of $\mathrm{H} 2 \mathrm{O}$ ). The mean changes in body weight of the WT and Cld-1 Tg mice were measured and graphically depicted. (B) Tumor burden was determined using colonoscopy, tumor multiplicity as assessed by gross visual inspection of the longitudinally opened colon. (C) Representative H\&E-stained images from AOM-DSStreated mice. The percentage of mice possessing dysplasia in the recovery protocol among WT and Cld- 1 Tg groups. Scale bar- $500 \mu \mathrm{m}$ and $100 \mu \mathrm{m}$ respectively. 
(A)

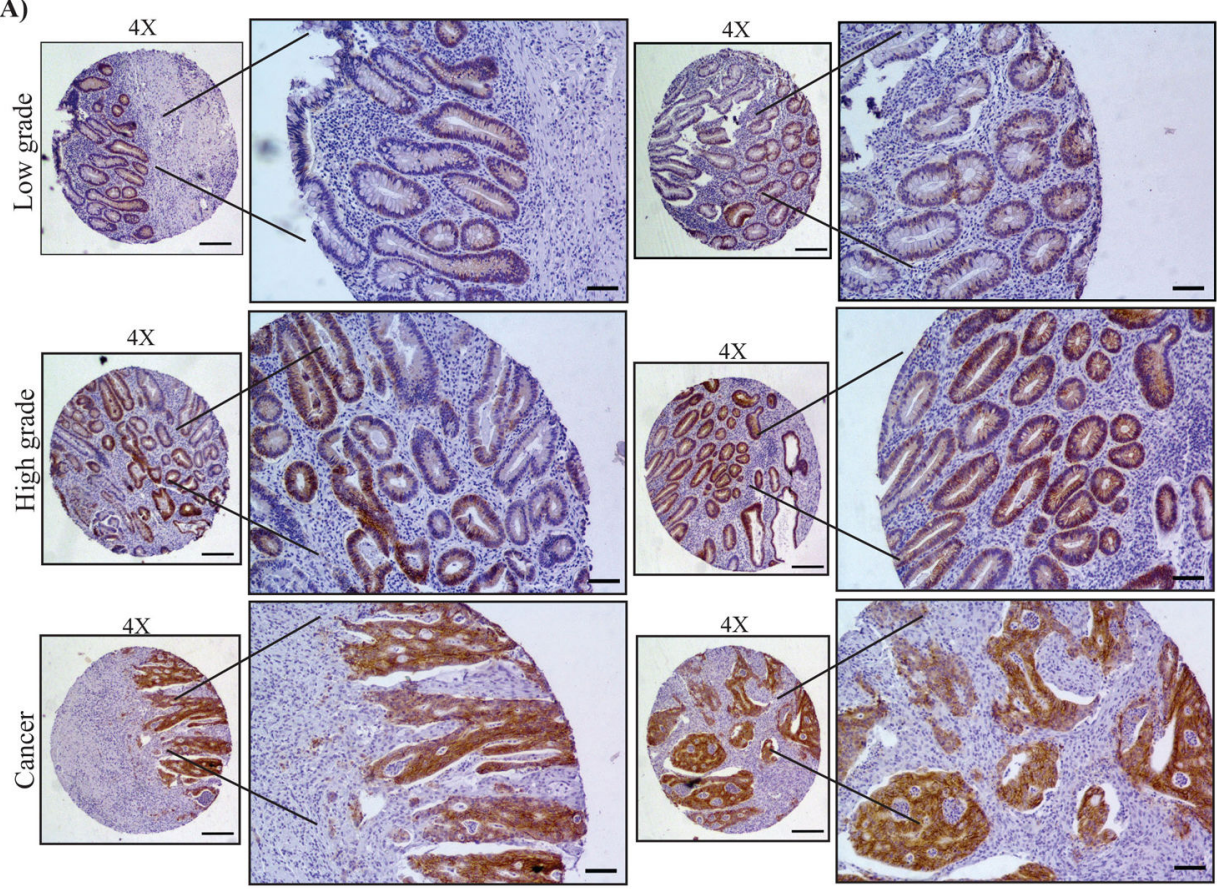

(B)

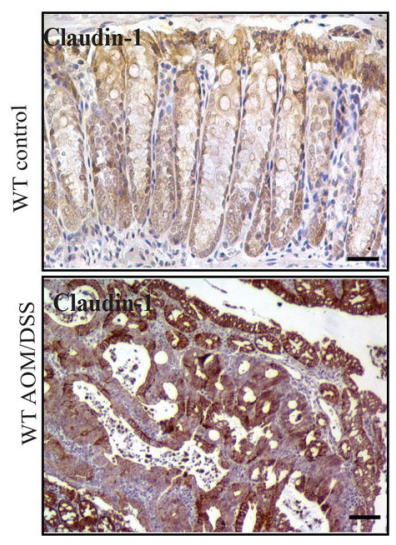

(C)
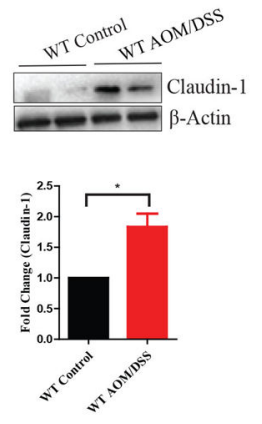

Fig. 3.

Comparative analysis of Cld-1 expression and subcellular localization during step wise progression to colon carcinogenesis in IBD patients:(A) Comparison of the immunohistochemical pattern of Cld-1 among the sequential stages (LG, HG and Cancer) towards the progression towards colon cancer from human IBD biopsies, the images show strong cytoplasmic and nuclear staining in colon cancer. The tissue sections of LG and HG dysplasia shows less intense expression of Cld-1 but significantly more than mild and moderate inflammatory colonic tissue sections. The square with enlarged image highlights the increased expression of Cld-1 (Magnification - 4X) Scale bar- $500 \mu \mathrm{m}$ and $200 \mu \mathrm{m}$ respectively. (B) Tissue sections from the colon of mice subjected to AOM/DSS protocol shows intense in Cld-1 staining than control mice. Scale bar is $100 \mu \mathrm{m}$. The inserts show the magnified pictures of the respective images. (C) Western blotting and its densitometry 
analysis for Cld-1 from the colon tumor of AOM/DSS mice and normal control of control mice. $\beta$-Actin was served as loading control. 
(A)
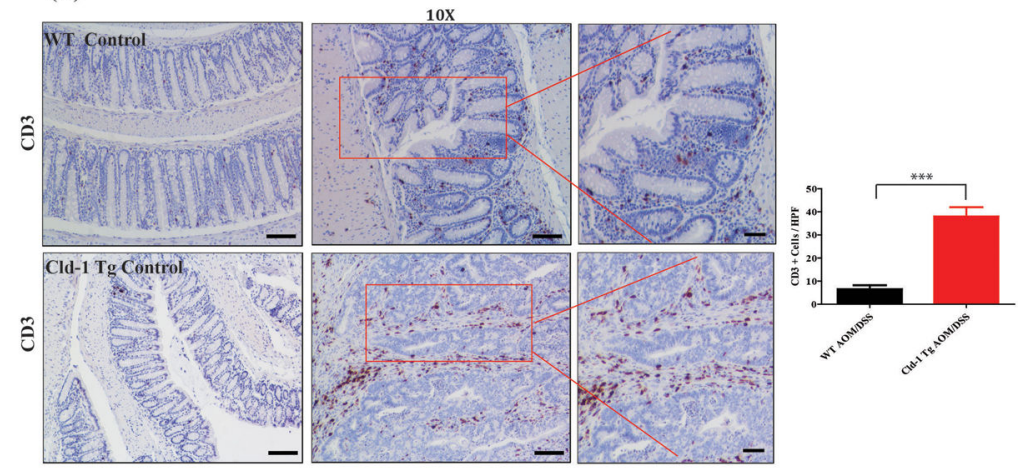

(B) F-480/iNOS/DAPI
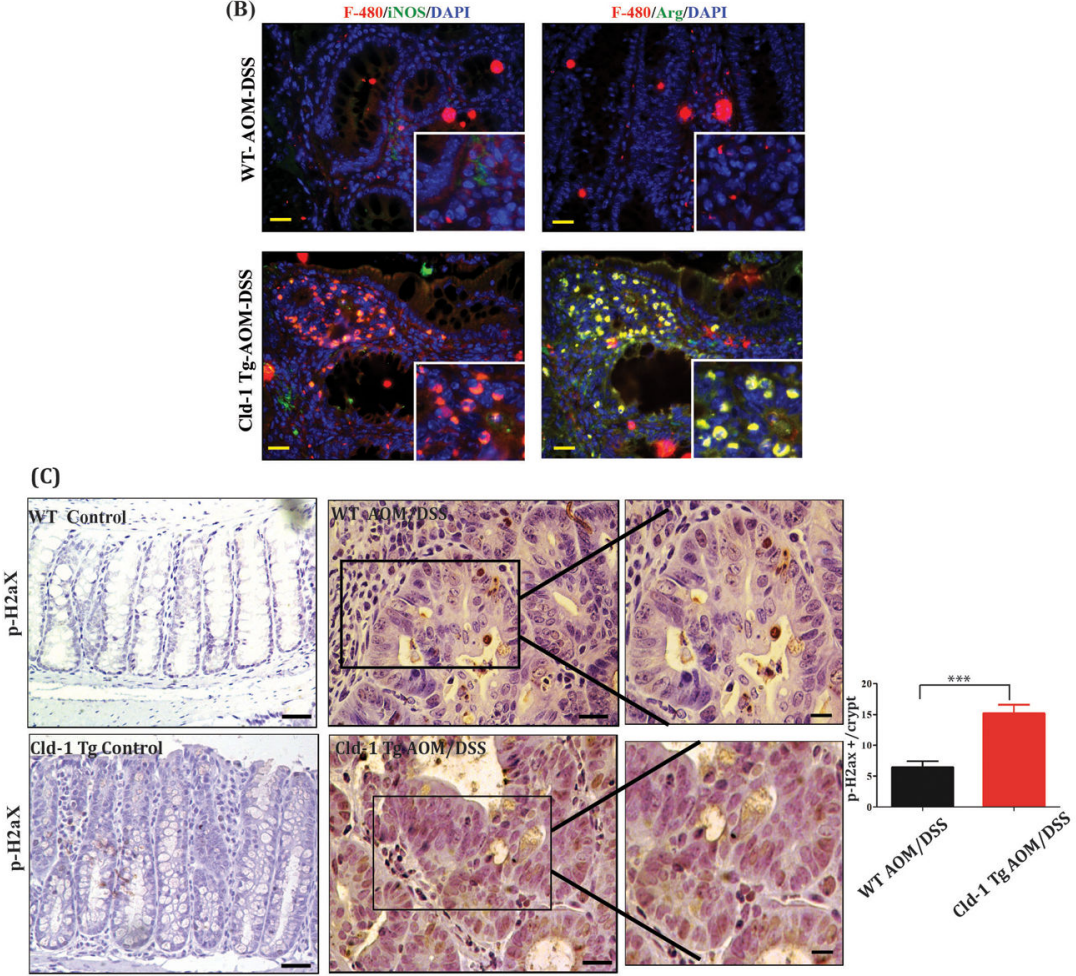

Fig. 4. Cld-1 Tg mice is associated with increased T-cell infiltration, M2 macrophage accumulation and intensive cellular damage in inflamed tumour environment:

(A) Representative CD3 T-cell population in the control and colonic tumor tissues section between WT and Cld-1 Tg AOM/DSS mice. The colour code for positive expression is brown and the counter stain is blue. (Magnification - 20X, Scale bar - $100 \mu \mathrm{m}$ ). (B)

Representative immunofluorescence images of F-480/iNOS and F-480/Arg in the tumoral colonic tissues sections of control and AOM/DSS mice (inserts shows enlarged images of the positive cells for F-480/iNOS and F-480/Arg) Scale bar- $50 \mu \mathrm{m}$.. (C) Representative images of the immunohistochemical staining for $\mathrm{p}-\mathrm{H} 2 \mathrm{AX}$ and quantification of number of $\mathrm{p}$ $\mathrm{H} 2 \mathrm{AX}$ positive cells in untreated and AOM/DSS groups across 20 random fields. The square with enlarged image highlights the increased expression of p-H2AX, Colour code: Brown protein expression, Blue - Hematoxylin counter stain, Results are statistically significant at $p<0.05$. Values sharing following symbols differ significantly, ${ }^{*}$ - compared with WT AOM/DSS. (Magnification - 20X, Scale bar - $100 \mu \mathrm{m}$ ). 

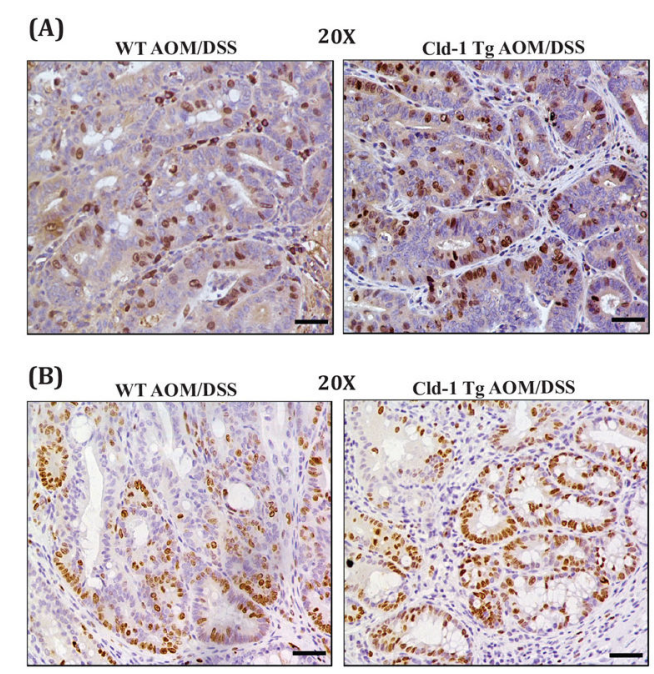

(C)
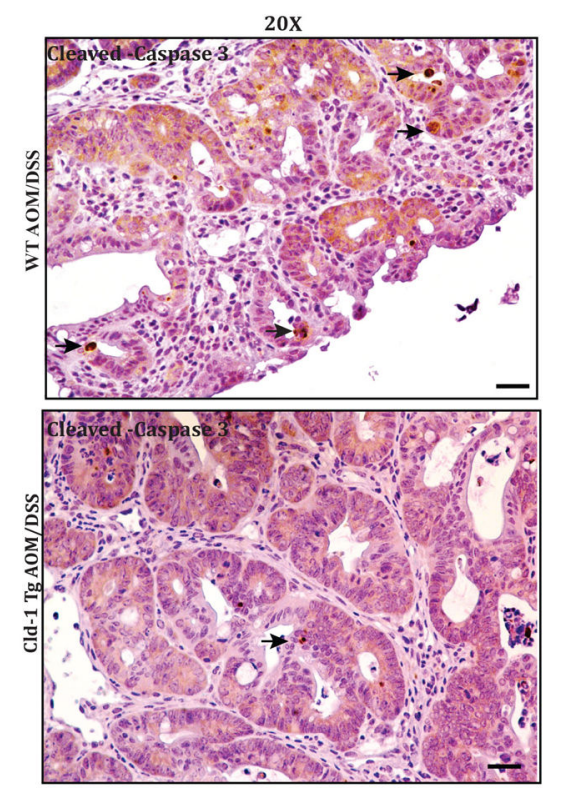
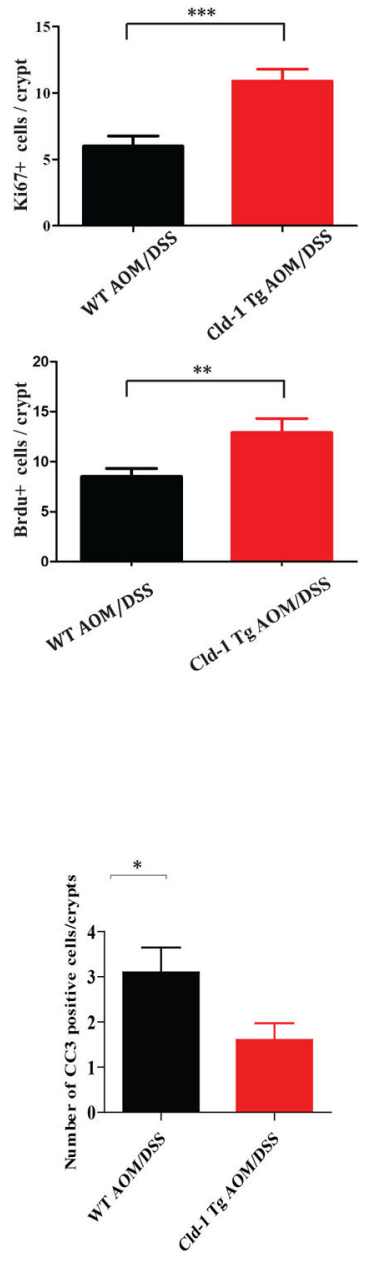

Fig. 5. Cld-1 strongly attenuates epithelial cell proliferation among AOM/DSS tumour:

(A) Representative photographs for the immunohistochemical staining of Ki67 in untreated, AOM/ DSS induced tumours in WT and Cld-1 Tg groups, quantification of number of positive cells across 20 random fields. (B) Brdu staining of the colonic sections of untreated and AOM/DSS induced groups in WT and Cld-1 Tg groups, graphical representation of the number of Brdu positive cells across 20 random fields. Results are statistically significant at $p<0.05$. Values sharing following symbols differ significantly, ${ }^{*}$ - compared with WT AOM/DSS. (Magnification - 20X, Scale bar - $100 \mu \mathrm{m}$ ). (C) Apoptotic cells were analysed by immunohistochemical staining of cleaved caspase-3 (CC3), graphical representation of the number of apoptotic cells across 20 random fields. Arrows indicate the epithelial cells positive for Cleaved caspase-3. The square with enlarged image highlights the increased expression of Cleaved Caspase-3, colour code: Brown - protein expression, Blue Hematoxylin counter stain, Scale bar $-100 \mu \mathrm{M}$. 


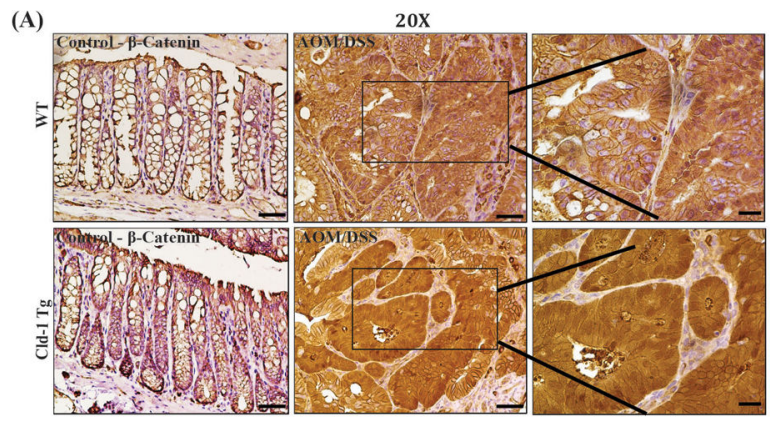

(B)
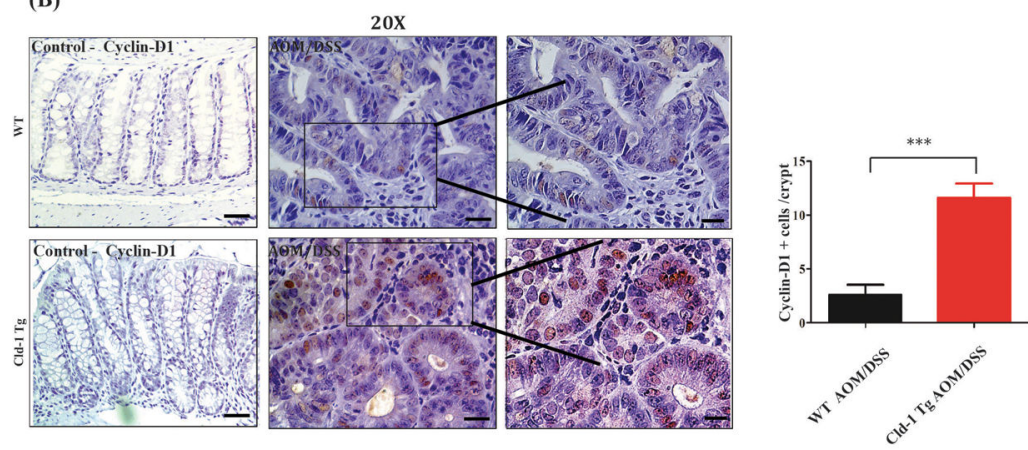

(C)

10x
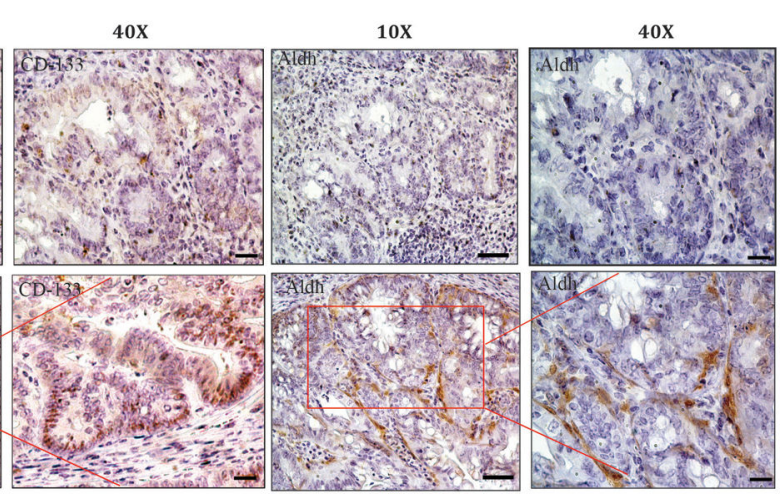

Fig. 6. $\beta$-catenin signalling is activated in Cld-1 Tg mice:

(A) Representative images of $\beta$-catenin in the colonic tissue sections of Control and AOM/DSS treated WT and Cld-1 Tg mice. The square with enlarged image highlights the significantly increased expression of $\beta$-catenin, colour code: Brown - protein expression, Blue - Hematoxylin counter stain, Scale bar - $100 \mu$ M. (B) The expression status of CyclinD1, a key downstream molecule of $\beta$-catenin signalling cascade as assessed by immunohistochemistry. The square with enlarged image highlights the significantly increased expression of Cyclin-D1, colour code: Brown - protein expression, Blue Hematoxylin counter stain, Scale bar - $100 \mu \mathrm{M}$. (C, D) Representative images of the colonic tissue sections stained for cancer stem cell markers, CD133 and Aldh. The square with enlarged image highlights the increased expression of CD133 and Aldh, colour code: Brown - protein expression, Blue - Hematoxylin counter stain (Magnification - 10X, Scale bar $100 \mu \mathrm{m})$. 
(A)

(B)
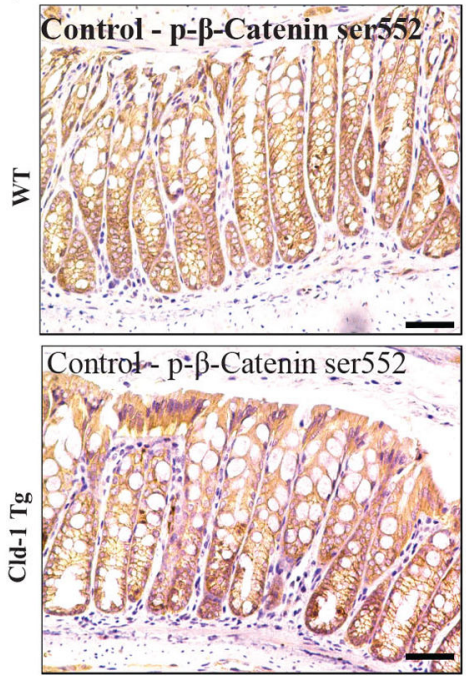

20X
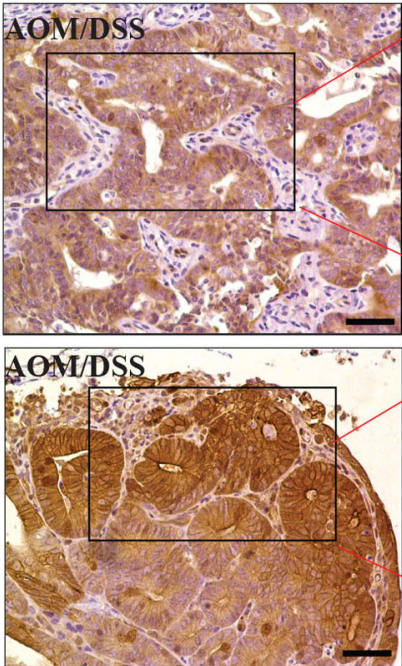
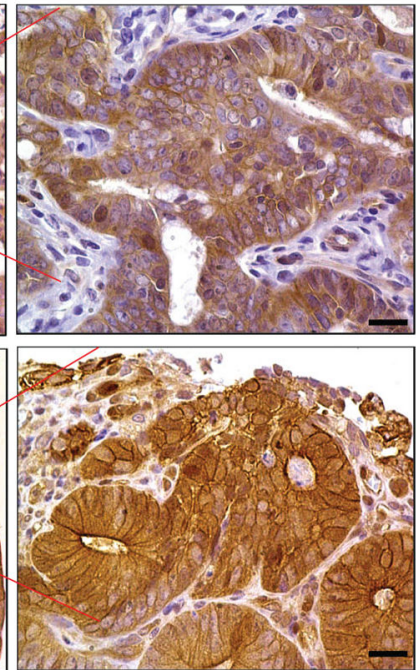
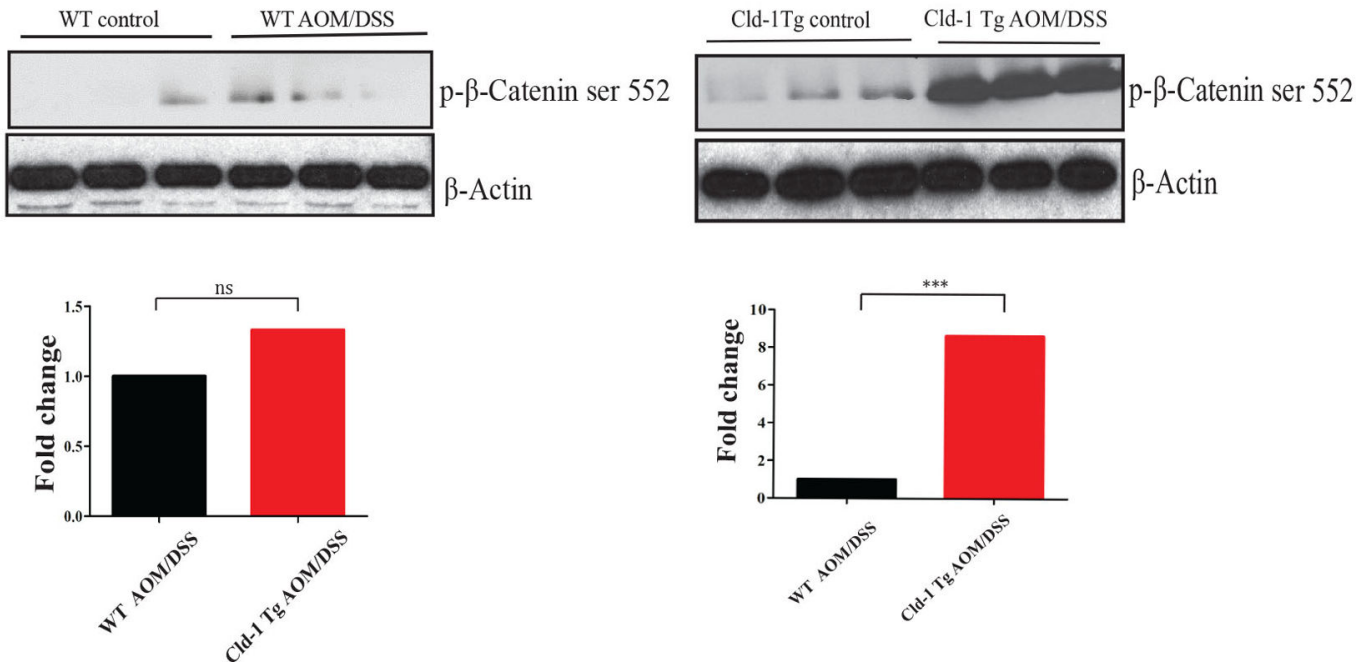

Fig. 7. Cld-1 Tg Mice shows enhanced p- $\beta$-CatSer-552 when subjected to AOM/DSS exposure: (A) Immunohistochemistry staining for $\mathrm{p}-\beta-\mathrm{Cat}{ }^{\mathrm{Ser}-552}$ in the colonic tissue sections of control and AOM/DSS group of mice in WT and Cld-1 Tg mice, Scale bar-100 $\mu \mathrm{m}$ respectively. (B) Immunoblot and densitometric analysis of p- $\beta-\mathrm{Cat}{ }^{\mathrm{Ser}-552}$ in colon tissue samples among control and AOM/DSS group of mice. Results are statistically significant at $p<0.05$. Values sharing following symbol differ significantly, ${ }^{*}-\mathrm{Vs}$ WT AOM/DSS. Each value is expressed as mean \pm S.E for three separate experiments $(n=3)$. 


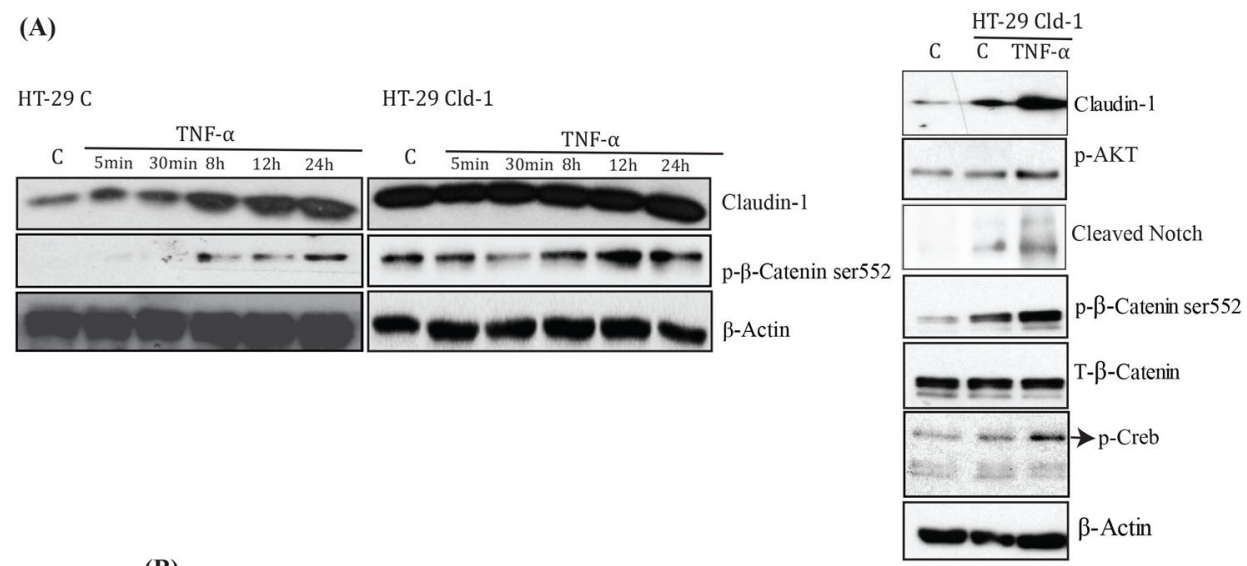

(B)

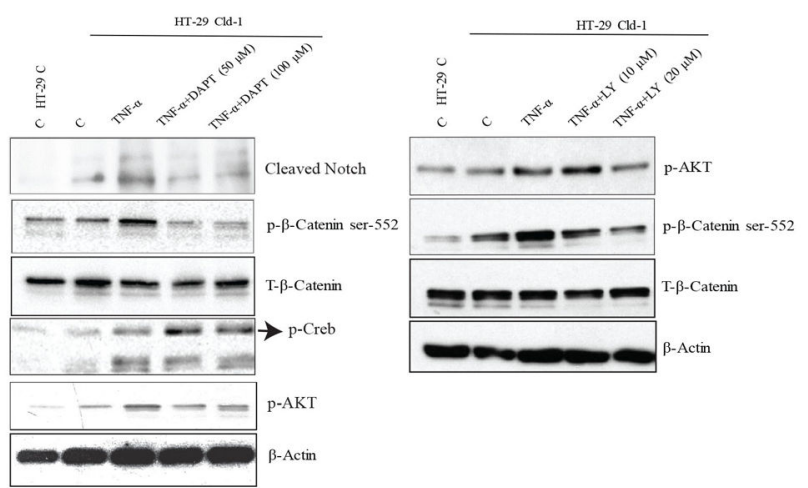

(C)
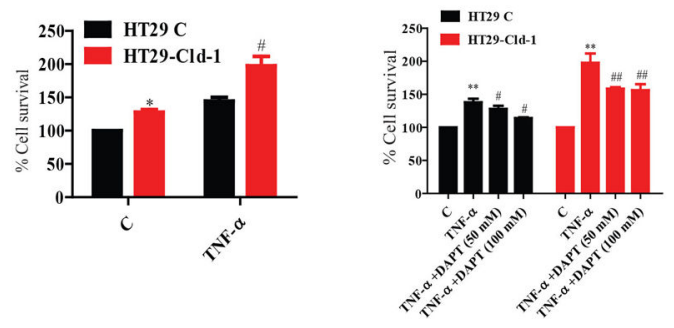

Fig. 8. Evidence that Notch and p-AKT are the crucial mediators of Cld-1 mediated activation of $\beta$-catenin:

(A) Immunoblot of p- $\beta$-Cat ${ }^{\mathrm{Ser}-552}$ in HT-29C IEC $^{\mathrm{C}}$ and HT-29Cld-1, IEC $^{\mathrm{Cld}-1}$ challenged with TNF-a $(10 \mathrm{ng} / \mathrm{ml})$ for $24 \mathrm{hrs}$. Western blotting analysis showing the up-regulation of $\mathrm{p}$ AKT and Cleaved Notch 1 with parallel increase in the accumulation of $p-\beta$-Cat ${ }^{\mathrm{Ser}-552}$ Immunoblot of HT-29C and HT-29 ${ }^{C l d-1}$ cells challenged with TNF-a with/without DAPT (50 or $100 \mu \mathrm{M})$ or LY294002 (10 and $20 \mu \mathrm{M})$. (C) TNF-a exposure attenuates the proliferation rate of HT-29C and HT-29 ${ }^{\mathrm{Cld}}{ }^{1}$ cells as evidenced by MTT assay, Results are statistically significant at $p<0.05$. Values sharing following symbol differ significantly, *Vs HT-29C, \# - Vs HT-29C exposed to TNF-a Each value is expressed as mean \pm S.E for three separate experiments $(n=3)$. The proliferation rate of HT-29C and HT-29Cld ${ }^{1}$ Under TNF-a with/without DAPT/ LY294002 as evidenced by MTT assay. Results are statistically significant at $p<0.05$. Values sharing following symbol differ significantly, * - Vs HT-29C, 
\# - Vs HT- $29^{\mathrm{C} / \mathrm{Cld}-1}$ with TNF-a. Each value is expressed as mean \pm S.E for three separate experiments $(n=3)$. 